\title{
The Brave New World of Epigenetics: Embracing Complexity in the Study of Speech and Language Disorders
}

\author{
Shelly Jo Kraft • Laura Segebart DeThorne
}

Published online: 26 June 2014

(C) Springer International Publishing Switzerland 2014

\begin{abstract}
The study of causal influences on marked disorders of speech and language have been dominated by dichotomies and reductionist models. However, the emergent evidence of epigenetics forces us to acknowledge the complex mechanisms that underlie such conditions. The purpose of this article is to highlight future directions for studying the causal mechanisms that underlie children's speech and language disorders, with an emphasis on speech sound disorders and specific language impairment. In doing so, we will briefly highlight past methods of studying genetic effects on children's speech sound and language disorder profiles and propose the dynamic systems theory as a meaningful framework for studying the complex landscape of childhood speech-language development. This model challenges traditional dichotomies between genetic and environmental effects and between beneficial versus deleterious effects/factors.
\end{abstract}

Keywords SLI $\cdot$ SSD $\cdot$ epigenetics $\cdot$ Developmental Systems Theory $\cdot$ Candidate genes

\section{Introduction}

Genetics offers a delimited but critical window into understanding the complex causal mechanisms associated with developmental speech and language disorders. Some isolated cases aside, recent findings have highlighted that specific

\footnotetext{
S. J. Kraft $(\bowtie)$

Communication Sciences and Disorders, Wayne State University, Rackham, 60 Farnsworth Street, Detroit, MI 48202, USA

e-mail: kraft@wayne.edu

L. S. DeThorne

Speech \& Hearing Science, University of Illinois at

Urbana-Champaign, 901 S. Sixth Street, Champaign, IL 61820, USA

e-mail: lauras@illinois.edu
}

language impairment (SLI) and speech sound disorders (SSD) do not follow single-gene Mendelian modes of transmission or causality [1-3]. These disorders, like many speech and language disorders, are now thought to result from multiplex interactions across various genetic and environmental factors. Traditional models and approaches toward etiology have been challenged by recent discoveries in the realm of epigenetic effects. Broadly, epigenetics refers to the study of influences on gene expression independent of direct changes in genetic code. Two key epigenetic mechanisms that influence DNA expression are methylation of DNA and modification of surrounding histone proteins. As succinctly summarized by Rice [4•], "The epigenome consists of chemical compounds that modify, or mark, the genome in a way that tells it what to do, where to do it, and when to do it. (p.1)" Such epigenetic processes govern cell differentiation, genetic imprinting, and X chromosome inactivation [5•, 6-8]. There is even evidence that some epigenetic markers are passed on to subsequent generations, broadening our concept of inheritance, and blurring traditional boundaries between genes and environments. In addition, epigenetics is likely to contribute to our understanding of the phenotypic variability associated with the same genotype, for example reduced penetrance, variable expressivity, and pleiotropy.

We are just beginning to understand how epigenetic processes contribute to differences in children's speech and language development; epigenetic processes have been implicated in cognitive development and developmental disorders such as autism, Fragile X, Angelman syndrome, and Rett syndrome $[5 \cdot, 9,10]$. For example, in most cases, Rett syndrome is associated with a mutation in the gene encoding $\mathrm{McCP} 2$, a methyl-DNA-binding protein involved in the silencing of methylated genes. Although the role of epigenetic effects on disorders such as SLI and SSD are less clear, Rice [4•] highlights ways in which the developmental phenotype of SLI is consistent with epigenetic influences, particularly in 
regard to age-related changes. In addition, Rice [4•] notes that a number of candidate genes associated with SLI are regulatory in nature (e.g., FOXP2, KIAA0319) and highlights the potential role of environmental factors, such as hormones and nutrition.

To accommodate the complexity of epigenetic effects, authors are beginning to situate genetic work within broader frameworks, such as the developmental systems theory (DST; e.g., Jaeger et al. [11•] and Müller et al. [12•]). DST acknowledges the arbitrary nature of genetic versus environmental boundaries and highlights the complex landscape of causal influences on child development. Specifically, DST stresses the point that cells, tissues, organs, organisms, and all other extra-organism levels of organization are fused in a fully coacting, mutually influential, and therefore dynamic system $[13$, p.10]. While different authors have underscored various tenets of DST [14, 15, 16•], key to the present paper is the concept that any current intrinsic state is a product of earlier developmental/contextual processes; consequently, how we define/divide nature versus nurture depends on the window through which we are looking. For example, Rice [4•] highlights the role of hormones and nutrition as traditional environmental factors that may impact language development through epigenetic effects. More specifically, Zeisel [17] outlines a mechanism in mice by which maternal diet during pregnancy results in epigenetic changes within the offspring that have permanent effects on hippocampus development and consequently, memory. These examples highlight the point that genetic and environmental effects are inextricably intertwined. Current states are always shaped by past patterns of interaction within and across organisms.

Using the dynamic systems framework as a guide, we intend to highlight the contributions and limitations of key methodological approaches to the genetic bases of developmental speech and language disorders, with a particular focus on SLI and SSD. In addition, we highlight four priorities for future directions.

\section{Contributions and Limitations of Various Approaches}

\section{Familial Aggregation Studies}

Formal examination of the tendency for speech and language difficulties to be shared by family members gained momentum in the 1980s through familial aggregation studies. Familial aggregation design begins through identification of probands, which in this case would be children with speechlanguage impairment, and control children considered to have typical speech-language development. If the probands demonstrate a higher rate of speech and language impairment within family members than control children, results support the tendency of the speech and language impairment to run in families. A review of seven familial aggregation studies of primary language disorders by Stromswold [18] found that the family incidence of language impairment in probands consistently exceeded that of controls; specifically, the median proband incidence was $35 \%$ across studies compared with $11 \%$ in control families (p.304). Similar patterns have also been reported specifically related to SSD [19]. In addition to revealing a consistent trend for speech-language impairments to run in families, familial aggregation studies have generally ruled out patterns of classic Mendelian inheritance including $\mathrm{x}$-linked transmission (see Hurst et al. [20] for a notable exception). As a result, familial aggregation studies have not been able to elucidate the mechanism through which speech and language impairments run in families, leaving open for debate the traditional distinctions between genetic versus environmental effects.

It is often assumed that disorders with complex inheritance patterns that cluster in a family must have a genetic contribution. While this is quite commonly the case, sometimes we can overestimate the genetic contribution, and sometimes there is not one. The role of common factors such as culture, diet, environmental exposure, or - most commonly - coincidence, with each familial aggregation must be assessed and considered. Ultimately, genetic contribution requires definitive proof with identified genes, loci, and alleles that are shared among affected individuals, and this can be a difficult task.

Complex diseases are often broken down into phenotypes that represent qualitative (aka discrete) and quantitative traits. Qualitative traits are those that are either present or absent. Quantitative traits are those that have some measurable attribute or can be present along a severity continuum. When related individuals are both affected by a trait, they are considered 'concordant'. Conversely, when they do not share a trait they are 'discordant'. Sometimes relatives can share a particular gene but still be discordant for the phenotypic disease trait. This commonly is the result of two individuals who do not share other factors that regulate that particular gene. The opposite can also occur, where two relatives share the same phenotypic trait but have vastly different genotypes in a multifactorial genetic disorder. So how do we parse apart the relative contributions of genes to complex diseases, and is it even possible?

\section{Twin Studies and Behavioral Genetic Methods}

Building upon familial aggregation designs, behavioral genetic methodologies promised a means to partition variance in a given trait, such as language skill, into genetic versus environmental effects based largely on predictable differences in genetic similarities across biological family members [21]. Using twin studies as a specific example, monozygotic (MZ) twins theoretically share $100 \%$ of their nonsegregating genes, whereas dizygotic (DZ) twins share on average only $50 \%$. 
Consequently, traits under partial genetic control should appear more similar in MZ than in DZ twins, and the extent to which zygosity can be used to predict similarity offers an estimate of genetic effects using intraclass correlations and model fitting procedures $[22,23]$. Concordance rates provide an index of how many twin pairs share a diagnosis such as speech or language impairment. A review of twin studies conducted by Stromswold [24] found consistently higher concordance rates for language impairments in $\mathrm{MZ}$ than in DZ twins, including studies focused on SLI (see also Lewis et al. [19], Plomin and Kovas [25]). Across ten studies, Stromswold [24] reported a mean concordance rate of $80 \%$ for MZ twins across studies, compared with a mean of $46 \%$ for DZ twins (p.659). Of particular interest to the present paper, the concordance amongst MZ twins is rarely $100 \%$, a finding consistent with such phenomena as reduced penetrance and epigenetic effects. Despite the intuitive appeal of behavioral genetic methodologies and their contribution in highlighting the influence of genes on children's speech and language development, they offer little insight into the specific genetic mechanism underlying inheritance, and they impose a gene versus environment dichotomy that oversimplifies the inherent complexity of causal processes $[13,26]$. In sum, "Familial aggregation studies, the analysis of twin concordance, and estimates of heritability do not specify which loci and alleles are involved, how many loci there are, or how a particular genotype and set of environmental influences interact to cause a disease or to determine the value of a particular physiological parameter. In most cases, all we can show is that there is a genetic contribution but little else" [27, pp. 158159].

However, epigenetic theories could explain the imperfect concordance rates. The integration of diverse environmental factors or stimuli, at any point in the brain's development, can alter the expression of genes, and result in different speech behaviors and traits in two genetically identical individuals. After all, speech and language disorders are today defined solely on the basis of atypical speech and language behaviors. Most of these differences typically develop over a period of time, and are not necessarily present at birth. Many disorders have a childhood onset between the ages of 2 and 6 years. "Epigenetic changes, including those that occur randomly during the highly complex process of brain development, could help explain the high discordance rates between monozygotic twins ... as well as the striking differences in prevalence for ... men versus women" [8, p. 65].

\section{Molecular Approaches}

Prior to the 1980 s, gene-disease discoveries were very rare. Advancements made in the last 35 years have made the identification of genetic determinants for disease phenotypes a daily occurrence. Currently, several molecular approaches usually fall under the umbrellas of association, linkage, or copy number variation studies. However, there is no standard molecular procedure that leads to the discovery of causative genes. Clues are used to guide the method based on phenotypic parallels with other disorders of known etiology (animal, insect, or human), heritability estimates, transmission models, known protein product, and any information available regarding potential chromosomal location or properties. Thankfully, geneticists have put together a large resource of databases with human genome mapping, expression data, and sequence data readily available to guide the molecular approach appropriate for each individual disorder of interest.

Association studies are basically a case-control design that sometimes takes into account the entire human genome as in a genome-wide association study (GWAS), to compare affected individuals with unaffected individuals who are matched for gender, age, and ethnicity. The strength of this approach is in the identification of common variants with small affect sizes (i.e. a small measure of potency for that particular allele) that contribute to a phenotype. These case-control studies can also be conducted within a single family that has a high number of affected individuals. In this case, the more closely related the individuals, the more genetic material they will have in common. As this increases the difficulty in identifying risk alleles, linkage studies are typically used in place of association studies when investigating with related populations.

Linkage studies utilize this relatedness coefficient as they investigate large sections of DNA that are passed across affected family members and are powered for identifying very rare variants with large-scale effects on the resulting phenotypic function of the individual. By using mathematical algorithms to calculate and control for relatedness, geneticists can determine much of what percent of the concordance can be predicted by relatedness and shared genes. However, this only partially clears the water, as the more closely related you are to another member of your family, the more of your environment you will share. Evidence of epigenetic effects highlights the need to expand our field's concept of environmental effects and underscores the reality that genes do not dictate phenotypic destinies. The study of copy number variation has developed from this understanding. For many genes, each person has a unique number of repeated genetic code that proportionately affects the amount of gene-product (protein) produced. Copy number variation is not a mutation, but a common contributor to traits that operate on a continuum of expression.

Across species, the translation of genes implicated in social behavior is influenced by such factors as social status, stressful events, maternal care, nutrition, hormones, and pheromones [4•, 28]. Similarly, genotypic variations associated with human cognitive development exhibit differential responses to environmental variables such as breast feeding, maltreatment, drug exposure, and low birth weight [29, 30]. Environmental influences play an enormous role in quantitative traits that 
vary from affected individual to affected individual. Most scientists now recognize that the majority of complex diseases are not caused by single genes with high penetrance and largescale deleterious effects on the gene product. Typically, a single gene is not sufficient to produce the associated disease/disorder phenotypes. More commonly, large networks of genes operate together, many times without known mutations but with common variants found in unaffected individuals, which when combined with environmental triggers are sufficient to alter phenotypes.

Once candidate regions of DNA have been recognized through methods of association, linkage, or copy number variant $(\mathrm{CNV})$ analysis, animal models are employed to solidify the phenotype-genotype relationship. Animal studies are particularly useful as we can control animal behavior in ways that we cannot in humans. We can utilize the accessibility and manipulability of animals that share genetic constellations with our own species to pinpoint certain genetically driven behaviors. Classically, the first association between a single gene and behavior was found in 1915 by Sturtevant, who saw that a mutation in the common fruit fly altered eye color and affected the mating behavior. Genes responsible for albino mice also result in less active and more cautious demeanor in open areas. These allelic associations, while outwardly appearing to be directly related, are being regulated by the environment. As eye pigment changes, so does the amount of light stimulation able to enter into the visual cortex. Altered stimulation and experiences of the same environment results in different behaviors and responses to that environment. The cause and effect diagram is no longer linear.

With many speech and language behaviors unable to be tested on animals, we instead search for probable candidates in humans. Like the word implies, scientists look for likely suspects with known alternations in functioning that have the potential to mitigate speech and language production. One of the most famous candidate genes thought to be responsible for SLI in a single family was FOXP2 identified in the KE family [20]. Located on the q arm of chromosome 7, FOXP2 is a brain-expressed transcription factor that affects brain development [31]. While further investigations of this early candidate have revealed its importance along the evolutionary timeline of language and communication [32, 33], it has not been shown to be responsible for the majority of individuals with SLI [34]. Other candidates have since been found (see Table 1 below), such as CNTNAP2, a gene also associated with autism [35]. More recently, SLI1 a region on chromosome 16 containing genes ATP2C2 and CMIP (also linked to autism [36]) and SLI2 on 19 [37, 38] were identified. $D Y X 8$, a region on chromosome 1 that demonstrates pleiotropy for SSD and dyslexia [39] along with aromatase (CYP19A1) located on chromosome 15 have been implicated. CYP12A1 is particularly interesting as it regulates estrogen synthesis in specific brain areas. It is related to synaptic plasticity and axonal growth [40]. Dyslexia, a disorder that commonly presents comorbidly with SLI and SSD [41], has been associated with ROBO1 and $\mathrm{ROBO} 2$ located on chromosome 3. These genes guide axons and influence neuronal axon growth. They were identified in a group of people with dyslexia in Finland [42]. KIAA0319, TTRAP, and DCDC2, all located on chromosome 6, disrupt neuronal migration and were identified in individuals with dyslexia by numerous research groups [43-45].

Finding candidate genes for speech and language disorders is an important step in the discovery process. However, as we have highlighted earlier, genes are not phenotypes. Genes are

Table 1 Summary of candidate genes associated with development speech and language disorders

\begin{tabular}{|c|c|c|c|c|c|}
\hline Gene & Function & Locus & Disorder & OMIM & Remarks \\
\hline FOXP2 & Transcription factor & $7 \mathrm{q} 31$ & SLI & 605317 & \\
\hline CNTNAP2 & $\begin{array}{l}\text { Cellular adhesion, receptors, potassium channel } \\
\text { localization, and cellular growth factors }\end{array}$ & $7 \mathrm{q} 35$ & SLI & 604569 & $\begin{array}{l}\text { Also implicated in Gilles de la Tourette } \\
\text { Syndrome, schizophrenia, epilepsy, } \\
\text { autism, ADHD, and MR }\end{array}$ \\
\hline $\mathrm{ATP} 2 \mathrm{C} 2$ & Hydrolysis of ATP and calcium transport & $16 \mathrm{q} 24.1$ & SLI & 613082 & Also linked to autism \\
\hline CMIP & $\begin{array}{l}\text { Signaling in the T-cell pathway and } \\
\text { phospholipid binding }\end{array}$ & $16 \mathrm{q} 23$ & SLI & 610112 & Also linked to autism \\
\hline DYX8 & Unknown & $1 p 36-34$ & SSD, dyslexia & 608995 & \\
\hline CYP19A1 & Synthesis of lipids and hormones & $15 \mathrm{q} 21.2$ & SSD, dyslexia & 107910 & \\
\hline KIAA0319 & Neuron guidance and migration in cerebral cortex & $6 \mathrm{p} 22.3$ & SLI, SDD & 609269 & Also implicated in reading disability \\
\hline ROBO1 & Axon guidance & $3 \mathrm{p} 12$ & Dyslexia & 602430 & \\
\hline ROBO2 & Axon guidance, molecular guidance & $3 \mathrm{p} 12.3$ & Dyslexia & 602431 & \\
\hline TTRAP & $\begin{array}{l}\text { Tumor necrosis factor, DNA binding and repair, } \\
\text { and magnesium ion binding }\end{array}$ & $6 \mathrm{p} 22.3$ & Dyslexia & 605764 & $\begin{array}{l}\text { Also known as TDP2. Also implicated } \\
\text { in narcolepsy }\end{array}$ \\
\hline $\mathrm{DCDC} 2$ & Embryonic neuron migration in the cerebral neocortex & $6 \mathrm{p} 22.1$ & Dyslexia & 605755 & Also implicated in reading disability \\
\hline
\end{tabular}

$A D H D$ attention-deficit hyperactivity disorder, MR mental retardation, OMIM Identifier in Online Mendelian Inheritance in Man (http://www.ncbi.nlm. nih.gov/sites/entrez?db=omim), $S D D$ speech sound disorders, $S L I$ specific language impairment, 
embedded within complex causal webs that extend outside the nucleus and beyond any individual organism.

\section{Conclusions: Future Directions}

Convergent findings across methodologies, emerging evidence of epigenetic effects, and implementation of a developmental systems framework offer at least four implications for future work. First, we need to broaden our view of environmental effects and recognize that such factors are not easily delineated from genetic proclivities. A recent review of 1,305 abstracts published in American Speech-Language-Hearing Association (ASHA) journals between 2003 and 2013 revealed 233 studies that directly addressed causal influences on child language development. Of those, $88 \%(192 / 233)$ focused on linguistic input and general family/caregiver descriptors such as SES [46•]. Very few studies, only $5 \%(12 /$ 233), examined such factors as nutrition, toxin exposure, stress, and hormones on child language development, even though we know from work in other fields that such factors are prominent in influencing child development. As an example, a study by Caspi et al. [30] found that the influence of early diet on the IQ of children was moderated by children's genotype. Specifically, early breast feeding was associated with a higher mean IQ of approximately 6 points for children with one particular allele. For children without that particular allele, early breast feeding was not associated with IQ differences. Such influences are just beginning to be explored in relation to developmental speech-language disorders (e.g., Mahurin-Smith and Abrose [47•]).

Second, we need to move away from single gene models of development speech-language disorders and toward common variant models of quantitative trait loci. If we use autism as an example: current sequencing studies are suggesting hundreds of genes (likely $>250$ ) to be associated with the disorder. These are genes that a majority of the unaffected population also carry, with individualized mutations and CNVs (Kraft, unpublished ongoing work). These are 'normal' and highly prevalent genetic variations operating in concert with each other, and, with the right degree of expression and distinctive cellular interaction, result in the social communication disorder that we recognize as autism. With this many players, one can imagine the nearly endless number of combinations possible to potentially result in the behavioral phenotype of autism. It makes sense that it is recognized and described as a spectrum disorder with behaviors that range from very mild to very severe. As of 2012, Linkage, GWAS, CNV, genomewide $\mathrm{CNV}$, and expression profile studies have compiled more than 2,193 candidate genes, 4,544 CNVs, and 158 linkage regions associated with autism [48•], all waiting to be replicated. When attempting to gain enough power to achieve replicable findings, the study size, homogeneity of the participant profiles, and the endless environmental interactions and modifications of DNA point to an ever-moving target - or approximately 250 targets. The standard approaches just cannot capture the behavior of these common variants.

Third, we need to move beyond the traditional dichotomy of genetic versus environmental effects to embrace full and complex webs of causal influences, including epigenetic effects. Studying such effects is likely to include new techniques and approaches as well as the combination of traditional methods. We most often look to advancements made in psychiatric disorders when contemplating the future of speech and language disorders. In the last 5 years, a method for investigating epigenetic mechanisms of gene expression through transcriptional mechanisms has been proposed using chromatin immunoprecipitation (ChIP) in psychiatry [8]. Using brain regions that are congruent in animal models or in postmortem human brains, researchers are able to detect the level of activation or inactivation of a given gene in that region [49, 8]. Using a combination of methods, Gregory et al. [9] utilized CNV and gene-expression analysis to examine the role of the oxytocin signaling pathway in autism. The authors identified a heterozygous deletion of the oxytocin receptor gene (OXTR) in an individual with autism and his mother (who did not have autism but possible obsessive-compulsive disorder [OCD]). The subject's brother with autism did not have the same deletion but appeared to have increased methylation of the gene in comparison with his father (who did not have autism). The authors also found converging evidence of 'hypermethylated' OXTR in group comparisons of individuals with autism compared with controls, using both peripheral blood cells and postmortem samples from the temporal cortex. Together, these results implicated the OXTR signaling pathway in the etiology of autism, including potential epigenetic differences in regulation. In sum, converging evidence across methodologies, paired with new techniques for studying gene expression, are likely to lead the way in understanding the underlying causal mechanisms for developmental speech and language disorders such as SLI and SSD.

Finally, the complexity of studying causal effects also invites us to re-examine the tendency to dichotomize specific factors, be they genetic or environmental, as positive or negative. In particular, there is a tendency in the literature on disorders to label specific genetic variations as 'bad or incorrect mutations,' presumably because the associated phenotypes are considered undesirable by those doing the research. However, in the grand evolutionary scheme of things, variation can be viewed as the fuel of success, and, at the individual level, the impact of allelic variation often cannot be considered independently from the context in which it is situated [50, 13]. In addition, the same gene may have antagonistic pleiotropic effects. Increasingly, phenotypes traditionally considered as disorders are being embraced as valuable forms of cultural- 
linguistic diversity [51, 52•]. Similar to genes, environmental influences on child language development, such as the nature of linguistic input, are often discussed as positive or negative, whereas the truth is often more complicated. As an example from animal models, research on maternal care in rats suggests that stressful living conditions are associated with less maternal nurturing behaviors (i.e., licking and grooming), which is thought to lead to more anxious pups through differences in gene expression in the reared offspring [7]. However, of particular interest, such anxiety may in fact be advantageous for the pups in the living conditions to which they were born. Griffiths and Tabery $\left[16^{\bullet}\right]$ capture this essence of socialization when they state "Organisms reproduce themselves by reproducing the context of development." (p. 81). The extent to which a given factor, such as parenting practice, is beneficial is often dependent on the larger environmental context. In sum, such complexity does not mean that certain environments and their associated traits are not going to prove problematic; indeed they likely will. However, taking a complex system approach to such disorders, and by virtue of association their etiology, creates a much broader and more flexible landscape for enacting positive change.

\section{Compliance with Ethics Guidelines}

Conflict of Interest Shelly Jo Kraft and Laura Segebart DeThorne declare that they have no conflict of interest.

Human and Animal Rights and Informed Consent This article does not contain any studies with human or animal subjects performed by any of the authors.

\section{Glossary}

Cell

differentiation

The epigenetic process by which the same DNA code is differentially expressed across distinct tissue types, coordinated in large part through control of chromatin structure.

Genetic The epigenetic process involving DNA imprinting methylation that crosses generations through the germline leading to differential expression of genetic material based on parental heritage (maternal or paternal). In the case of a maternally imprinted gene, the copy inherited from the mother is active while the copy from the father is silenced. In a paternally imprinted gene, the situation is reversed.

Pleiotropy The association of one gene with multiple phenotypic traits that seem unrelated. Antagonistic pleiotropy refers to the reality that a single gene can lead to both beneficial and detrimental phenotypic effects.

Reduced Reference to the fact that an autosomal penetrance dominant gene does not produce the associated phenotype in all organisms that carry the gene; $75 \%$ penetrance indicates that $75 \%$ of organisms that carry the gene would be expected to demonstrate the trait of interest.

Variable Variation in the degree to which a expressivity genotype is phenotypically expressed across individuals.

$\mathrm{X}$ chromosome The epigenetic process involving DNA inactivation methylation that deactivates one copy of the $\mathrm{X}$ chromosome in each cell.

\section{References}

Papers of particular interest, published recently, have been highlighted as:

- Of importance

1. Bishop DVM, Hayiou-Thomas ME. Heritability of specific language impairment depends on diagnostic criteria. Genes Brai Behavior. 2008;7:365-72.

2. Caylak, E. (2011). Biological/Biochemical features and molecular genetics of specific language impairment (SLI). In: eLS. John Wiley \& Sons Ltd, Chichester. http://www.els.net. doi: 10.1002/ 9780470015902.a0023688

3. Tallal P, Hirsch LS, Raelpe-Bonilla T. Familial aggregation in specific language impairment. J Speech Lang Hear Res. 2001;44: 1172-82.

4. Rice ML. Toward epigenetic and gene regulation models of specific language impairment: looking for links among growth, genes, and impairments. J Neurodevelopmental Disorders. 2012;4(27):1-15. This article appeared within a special issue focused on epigenetics in the study of the etiology of language, speech, and reading impairments. It is one of the first publications to discuss epigenetics as applicable to SLI, particularly in regard to elements of grammatical development across the school-age years. Specifically, the author proposes a growth-signaling disruption hypothesis to characterize SLI.

5. Day JJ, Sweatt JD. Epigenetic models in cognition. Neuron. 2011;70:813-29. This article offers a specific description of how epigenetic processes within postmitotic neurons may be contributing to learning and memory, particularly through a 'histone code.' The authors also touch upon epigenetically based disorders related to speech-language development, such as Rett syndrome, and offer examples of novel therapeutic interventions.

6. Kramer JM, van Bokhaven. Genetic and epigenetic defects in mental retardation. Int J Biochem Cell Biol. 2009;41:96-107.

7. Meaney MJ. Epigenetics and the biological definition of gene $x$ environment interactions. Child Dev. 2010;81(1):41-79.

8. Nestler EJ. Epigenetic mechanisms in psychiatry. Biol Psychiatry. 2009;65:189-90.

9. Gregory SG, Connelly JJ, Towers AJ, et al. Genomic and epigenetic evidence for oxytocin receptor deficiency in autism. BMC Med. 2009; 7:1-13. 
10. Monteggia LM, Kavalali ET. Rett syndrome and the impact of $\mathrm{MeCP} 2$ associated transcriptional mechanisms on neurotransmission. Biol Psychiatry. 2008;65(3):204-10.

11. Jaeger J, Irons D, \& Monk N The inheritance of process: A dynamical systems approach. J Exp Zool (Mol. Dev. Evol.), 2012; 591612. This article provides a rate illustration of dynamical systems approach using a toggle switch model of two genes ( $\mathrm{gt}$ and $\mathrm{Kr}$ ) that express transcription factors within the fruit fly.

12. Müller U, Baker L, Yeung E. A Developmental Systems approach to executive function. Adv Child Dev and Behav. 2013;45:39-66. This publication summarizes evidence that executive functioning is not immutable despite the fact it is highly heritable. The authors refer to epigenetics and DST.

13. Lerner, R.M. (2007). Developmental science, developmental systems, and contemporary theories and human development. In W. Damon \& R.M. Lerners (Eds.), Handbook of Child Psychology (Vol.1). (pp. 1-17). Retrieved from http://onlinelibrary.wiley.com/ doi/10.1002/9780470147658.chpsy0101

14. Thelen H. Dynamic systems theory and complexity of change. Psychoanal Dialogues. 2005;15(2):255-83.

15. Robert, J.S. (2003). Developmental systems theory. In Keywords and concepts in evolutionary developmental biology. Retrieved from http://proxy2.library.edu/login?url/=login?qurl=http://search. credoreference.com/content/entry/hupedb/developmental_ systems_theory $/ 0$

16. Griffiths PE, Tabery J. Developmental systems theory: What does it explain, and how does it explain it? Adv Child Dev Behav. 2013;44: 65-94. This online chapter challenges traditional notions in behavioral genetics and nativist cognitive psychology by highlighting core concepts of DST, including its historical development. Particularly relevant for language development, the authors highlight the critical role of socialization as closely entwined with inheritance, together serving the critical function of neural plasticity.

17. Zeisel SH. Epigenetic mechanisms for nutrition determinants of later health outcomes. Am J Clin Nutr. 2009;89:1488S-93.

18. Stromswold K. Genetics of spoken language disorders. Hum Biol. 1998;70(2):297-324.

19. Lewis BA, Shriberg LD, Freebairn LA, et al. The genetic bases of speech sound disorders: Evidence from spoken and written language. J Speech, Lang, and Hear Res. 2006;49:1294-312.

20. Hurst JA, Baraitser M, Auger E, Graham F, Norell S. An extended family with a dominantly inherited speech disorder. Dev Med Child Neurol. 1990;32:347-55.

21. Plomin R, DeFries JC, McClearn GE, McGuffin P. Behavioral genetics. 5th ed. New York: Worth; 2008. p. 305-33.

22. DeThorne LS, Hart SA. Use of twin design to examine evocative gene-environment effects within a conversational context. Eur J Dev Sci. 2009;3(2):175-94.

23. Purcell S. Statistical methods in behavioral genetics. In: Plomin R, DeFries JC, McClern GE, McGuffin P, editors. Behavioral Genetics. 5th ed. New York: Worth; 2008. p. 359-410.

24. Stromswold K. The heritability of language: A review and metaanalysis of twin, adoption, and linkage studies. Language. 2001;77(4):647-723.

25. Plomin R, Kovas Y. Generalist genes and learning disabilities. Psychol Bull. 2005;131(4):592-617.

26. Stromswold $\mathrm{K}$. The genetics of speech and language impairments. N Engl J Med. 2008;359:2381-3.

27. Nussbaum R, McInnes RR, Willard HF. Familial Aggregation. In Thompson \& Thompson Genetics in Medicine $-7^{\text {th }}$ ed. Elsevier Health Sciences. Philadelphia: Pennsylvania; 2007.

28. Robinson GE, Fernald RD, Clayton DF. Genes and social behavior. Science. 2008;322(5903):896-900.

29. Caspi A, Moffitt TE. Gene-environment interactions in psychiatry: Joining forces with neuroscience. Nat Rev Neurosci. 2006;7:583-90.
30. Caspi A, Williams B, Kim-Cohen J, et al. Moderation of breastfeeding effects on the IQ by genetic variation in fatty acid metabolism. PNAS. 2007;104(47):18860-5.

31. Liegeois F, Baldeweg T, Connelly A, et al. Language fMRI abnormalities associated with FOXP2 gene mutation. Nat Neurosci. 2003;6:1230-7.

32. Enard W, Przeworki M, Simon FE, et al. Molecular evolution of FOXP2, a gene involved in speech and language. Nature. 2002;418:869-72.

33. Wade, N. (2003). Early voices: The leap to language. New York Times, July 15 ed.

34. Maeburn E, Dale PS, Craig IW, Plomin R. Language-impaired children: No sign of the FOXP2 mutations. NeuroReport. 2002;13(8):1075-7.

35. Vernes SC, Phil D, Newbury DF, et al. A functional genetic link between distinct developmental language disorders. N Engl J Med. 2008;359(22):2337-45.

36. Van der Aan N, Vandeweyer G, Reyniers D, et al. Haploinsufficiency of CMIP in a girl with autism spectrum disorder and developmental delay due to a de novo deletion on chromosome 16q23.2. Autism Res. 2012;5(4):277-81.

37. Newbury DF, Winchester L, Addis L, et al. CMIP and ATP2C2 Modulate Phonolical Short-Term Memory in Language Impairment. Am J Hum Genet. 2009;85:264-72.

38. The SLI Consortium. A genomewide scan identifies two novel loci involved in specific language impairment. Am J Hum Genet. 2002;70:384-98.

39. Miscimarra L, Stein C, Millard C, et al. Further evidence of pleiotropy influencing speech and language: Analysis of the DYX8 region. Hum Hered. 2007;63(1):47-58.

40. Anthoni H, Sucheston LE, Lewis BA, et al. The aromatase Gene CYP19A1: Several genetic and functional lines of evidence supporting a role in reading, speech and language. Behav Gen. 2012;42:509-27.

41. Catts HW. Language impairments and reading disabilities. In: Kent $\mathrm{RD}$, editor. The MIT encyclopedia of communication disorders. Cambridge, MA: MIT Press; 2004. p. 329-31.

42. Nopola-Hemmi J, Myllyluoma B, Haitia T, et al. A dominant gene for developmental dyslexia on chromosome 3. J Med Genet. 2001;38:658-64.

43. Grigorenko EL, Wood FB, Meyer MS, Pauls DL. Chromosome 6p influences on different dyslexia-related cognitive processes: Further confirmation. Am J Hum Genet. 2000;66(2):715-23.

44. Rice ML, Smith SD, Gayan J. Convergent genetic linkage and association to language, speech and reading measures in families of probands with Specific Language Impairment. J Neurodev Disord. 2009;1(4):264-82.

45. Smith SD. Genes, language development, and language disorders. Lang Commun. 2007;13(1):96-105.

46. Rogers C, Nulty K \& DeThorne L. J Rev Causal Influences on Child Lang Dev 2013. Poster presented at the Undergraduate Research Symposium, Champaign, IL. This study highlights the relatively exclusive focus on linguistic input as a causal factor in the study of childhood language disorders, at least within the field of communication sciences and disorders. The authors note the need to expand the field's view of environmental influences and diversify the study of nonlinguistic influences such as nutrition, toxins, illness, etc.

47. Mahurin-Smith J, Ambrose NG. Breastfeeding may protect against persistent stuttering. J Comm Disord. 2013;46:351-60. This article is the first published on breast feeding and a communication disorder and was highly recognized for its innovation. It discusses metabolic influence on brain development from an evolutionary and nutrient standpoint.

48. Xu L-M, LI J-R, Huange Y, Zhao M, Tang X, Wei L. AutismKB: An evidence-based knowledgebase of autism genetics. Nucleic 
Acids Research. 2012;40(D1):D1016-22. This article uses network analysis to compile data from over 30 databases to compile a review and analysis of literature regarding genetic implications in autism.

49. Akbarian S, Huang H. Epigenetic regulation in human brainFocus on histone lysine methylation. Biol Psychiatry. 2009;65: 198-203.

50. Järvilehto, T., \& Lickliter, R. (2009). Behavior: Role of genes. In: Encyclopedia of Life Sciences (ELS). John
Wiley \& Sons, Ltd: Chichester. doi: 10.1002/ 9780470015902.a0006181.pub2

51. Bagatell N. From cure to community: Transforming notions of autism. ETHOS. 2010;38(1):33-55.

52. Lane H, Pillard RC. The People of the Eye: Deaf Ethnicity and Ancestry. New York: Oxford University Press; 2011. p. 3-32. This book makes the case for deafness as an ethnicity rather than a disability, including historical information on deaf education, civil rights, and causality. 